\title{
Sprouty-2 regulates HIV-specific T cell polyfunctionality
}

\author{
Yen-Ling Chiu, $, 1,2,3,4$ Liang Shan, ${ }^{2}$ Hailiang Huang, ${ }^{5}$ Carl Haupt, ${ }^{1,2,3}$ Catherine Bessell,, 1,3 \\ David H. Canaday, ${ }^{6,7}$ Hao Zhang, ${ }^{8}$ Ya-Chi Ho, ${ }^{2}$ Jonathan D. Powell, 9 Mathias Oelke, 1,2,3 \\ Joseph B. Margolick, ${ }^{8}$ Joel N. Blankson, ${ }^{2}$ Diane E. Griffin, ${ }^{8}$ and Jonathan P. Schneck ${ }^{1,2,3}$
}

\begin{abstract}
${ }^{1}$ Department of Pathology, ${ }^{2}$ Department of Medicine, and ${ }^{3}$ Institute for Cell Engineering, Johns Hopkins University School of Medicine, Baltimore, Maryland, USA. ${ }^{4}$ Department of Medicine, Far-Eastern Memorial Hospital, New Taipei City, Taiwan. ${ }^{5}$ Department of Human Genetics, Johns Hopkins University School of Medicine, Baltimore, Maryland, USA. ${ }^{6}$ Cleveland VA GRECC, and ${ }^{7}$ Case Western Reserve University School of Medicine, Case Western Reserve University,

Cleveland, Ohio, USA. ${ }^{8}$ W. Harry Feinstone Department of Molecular Microbiology and Immunology, Johns Hopkins Bloomberg School of Public Health, Baltimore, Maryland, USA. ${ }^{9}$ Department of Oncology, Johns Hopkins University School of Medicine, Baltimore, Maryland, USA.
\end{abstract}

\begin{abstract}
The ability of individual $\mathrm{T}$ cells to perform multiple effector functions is crucial for protective immunity against viruses and cancer. This polyfunctionality is frequently lost during chronic infections; however, the molecular mechanisms driving $T$ cell polyfunctionality are poorly understood. We found that human $T$ cells stimulated by a high concentration of antigen lacked polyfunctionality and expressed a transcription profile similar to that of exhausted $\mathrm{T}$ cells. One specific pathway implicated by the transcription profile in control of $T$ cell polyfunctionality was the MAPK/ERK pathway. This pathway was altered in response to different antigen concentrations, and polyfunctionality correlated with upregulation of phosphorylated ERK. $T$ cells that were stimulated with a high concentration of antigen upregulated sprouty-2 (SPRY2), a negative regulator of the MAPK/ERK pathway. The clinical relevance of SPRY2 was confirmed by examining SPRY2 expression in HIV-specific T cells, where high levels of SPRY2 were seen in HIV-specific T cells and inhibition of SPRY2 expression enhanced the HIV-specific polyfunctional response independently of the PD-1 pathway. Our findings indicate that increased $S P R Y 2$ expression during chronic viral infection reduces $T$ cell polyfunctionality and identify SPRY2 as a potential target for immunotherapy.
\end{abstract}

\section{Introduction}

A growing consensus indicates that $T$ cells capable of simultaneously producing multiple effector functions, referred to as "polyfunctional" $T$ cells, are a key subset in the development of effective immune responses against pathogens and cancer (1-7). These T cells, in addition to producing cytokines such as IL-2, TNF- $\alpha$, and IFN- $\gamma$, produce chemokines and also display cytolytic function. In contrast to acute infection, optimal polyfunctional memory $\mathrm{T}$ cell responses are lost in chronic infections and cancer, both in humans and mice $(5,8,9)$. These cells have been characterized as "exhausted" $(8,10)$ and factors promoting the development of $\mathrm{T}$ cell exhaustion include persistent and high levels of antigen stimulation $(11,12)$, DC inhibition (3), and upregulation of inhibitory receptors, such as PD-1, on T cells (13-17). While recent work has highlighted a role for PD-1 and other inhibitory receptors in $\mathrm{T}$ cell exhaustion, blockade of inhibitory receptor signaling in HIV-specific T cells had only a moderate effect in reversing the exhaustion phenotype and increasing $\mathrm{T}$ cell polyfunctionality $(14,15)$, thus indicating that additional molecular mechanisms are involved in the inhibition of $\mathrm{T}$ cell polyfunctionality.

Differences in $\mathrm{T}$ cell polyfunctionality correlate with differences in memory $\mathrm{T}$ cell formation in response to immunization as well as to viral infection. Regulation of vaccine-induced adaptive immune

Conflict of interest: Under a licensing agreement between NexImmune and the Johns Hopkins University, Jonathan P. Schneck and Mathias Oelke are entitled to shares of royalties received by the University on sales of aAPC products described in this article. They also own NexImmune stock, which is subject to certain restrictions under University policy. Jonathan P. Schneck is a member of the company's Board of Directors and Scientific Advisory Board. Jonathan P. Schneck also has a sponsored research agreement with Miltenyi Biotec. The terms of these arrangements are being managed by the Johns Hopkins University in accordance with its conflict of interest policies. Citation for this article: J Clin Invest. 2014;124(1):198-208. doi:10.1172/JCI70510. responses is complex and, in part, dependent on antigen dose (1). In animal models, high-dose vaccination results in inferior $\mathrm{T}$ cell polyfunctionality, poor memory formation and weaker immune protection as compared with optimal dose vaccination $(4,18)$. As a result, polyfunctional $\mathrm{T}$ cells are more than just a "marker" of protective immune response. The molecular mechanisms linking high-dose antigenic stimulation with inferior polyfunctionality and poor memory formation remain largely unclear.

Collectively, these data indicate the need to understand the molecular control of $\mathrm{T}$ cell polyfunctionality, especially in the context of antigen dose. Elucidating the molecular details underlying $T$ cell polyfunctionality could provide additional insights into $\mathrm{T}$ cell exhaustion and serve as a basis for vaccine design by optimizing $\mathrm{T}$ cell activity against virus infection or cancer.

In this study, we investigated the control of polyfunctionality in primary human virus-specific $\mathrm{CD} 8^{+} \mathrm{T}$ cells in response to antigen. In our model system, memory influenza-specific $\mathrm{T}$ cells repetitively stimulated with a high antigen concentration, $10 \mu \mathrm{M}$ of the influenza M1 peptide on monocyte-derived DCs (moDCs), showed robust influenza antigen-specific CD8 ${ }^{+}$ $\mathrm{T}$ cell proliferation, but low levels of polyfunctionality. In contrast, an optimal antigen concentration was determined, which induced highly polyfunctional influenza-specific T cells. An antigen concentration-dependent effect on polyfunctionality could also be demonstrated in naive human $\mathrm{CD}^{+} \mathrm{T}$ cells. Genomic gene set enrichment analysis (GSEA) revealed that the global transcriptome of high antigen concentration-induced $\mathrm{T}$ cells was similar, but not identical, to that of exhausted $\mathrm{T}$ cells observed in chronic infections. Importantly, low-level polyfunctionality induced by high antigen concentration stimulation led to increased expression of inhibitory receptors without evidence 

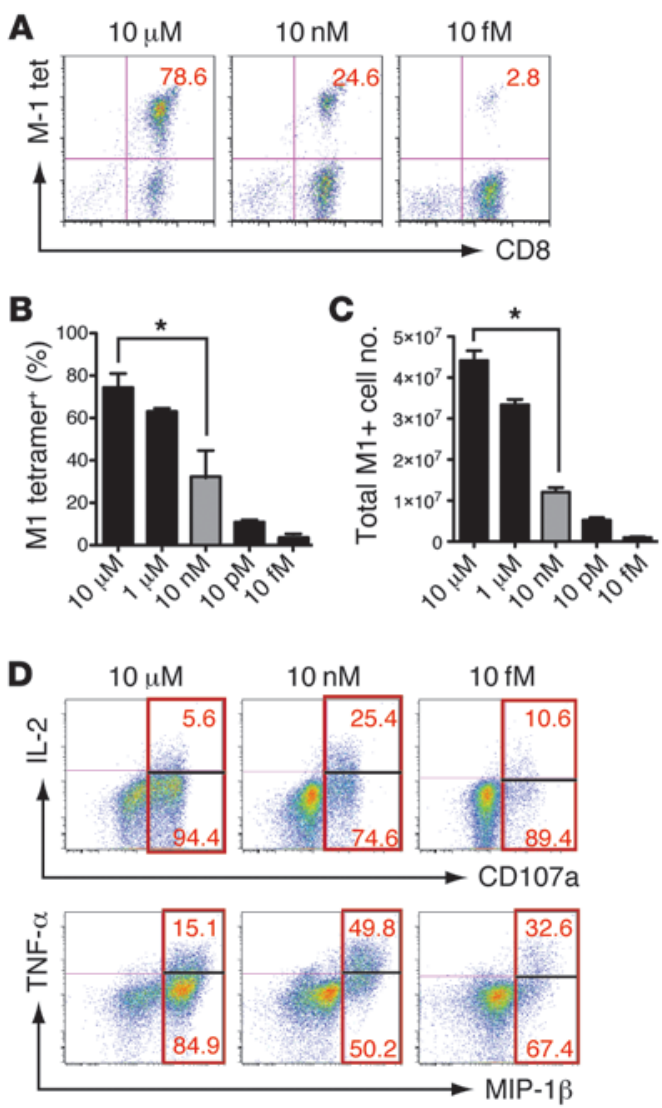

$\mathbf{E}$

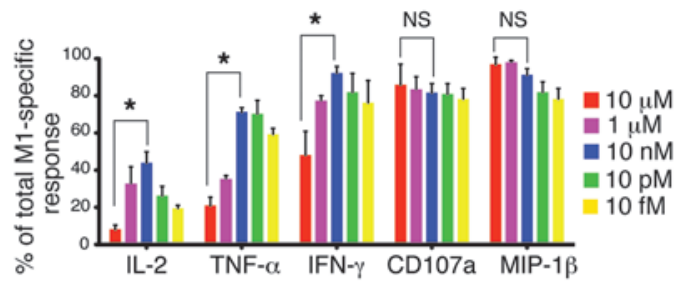

$\mathbf{F}$

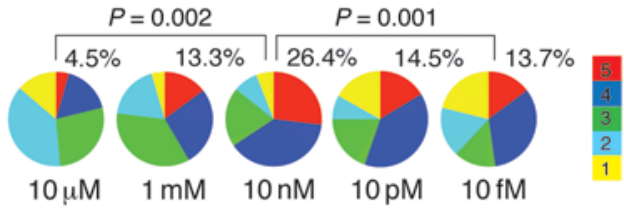

G
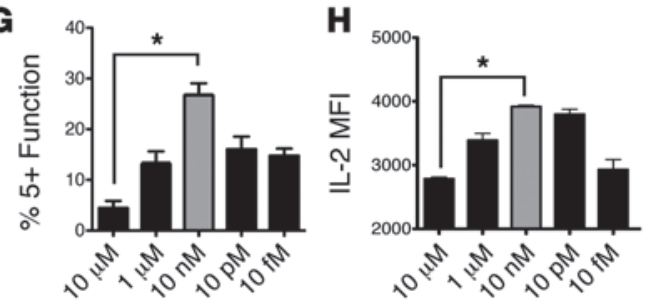

Figure 1

Influenza virus M1-pulsed moDCs induce concentration-dependent proliferation of M1-specific CD8 ${ }^{+} T$ cells with variable levels of polyfunctionality. (A-C) Percentage of M1-specific T cells and M1-specific T cell expansion. Autologous moDCs pulsed with variable amounts of the HLA$A^{*} 0201$ restricted immunodominant M1 peptide $(10 \mu \mathrm{M} \sim 10 \mathrm{fM})$ were used to stimulate HLA-A*A201+CD8+ $T$ cells ex vivo weekly for 2 weeks. Cultures were analyzed on D14. (D-H) Functional assessment of M1-specific T cells: polyfunctionality assessment of M1-specific T cells reveals significant impairment of T cells induced with a high antigen concentration (10 $\mu \mathrm{M}$ peptide-pulsed moDCs). (D) Top row: intracellular cytokine staining for IL-2 versus CD107. Numbers in red represent the percentage of IL-2-producing (right upper quadrant) versus IL-2-negative cells (right lower quadrant) in the CD107a-positive cell population. Bottom row: intracellular cytokine staining for TNF- $\alpha$ versus MIP-1 $\beta$. Numbers in red represent the percentage of TNF- $\alpha$-producing (right upper quadrant) versus TNF- $\alpha$-negative cells (right lower quadrant) in the MIP-1 $\beta$ positive cell population. (E) Percentage of individual effector function expression out of total antigen specific response. (F) Polyfunctionality pie charts. Each slice of pie represents the percentage of cells expressing between 1 and 5 effector functions. To compare different pie charts, permutation test with 10,000 repetitions was used. (G) The percentage of $5+$ function cells in each T cell culture. (H) Amount of IL-2 produced by $5+$ function cells. IL-2 production was quantified by measuring MFI and plotted for $5+$ function cells. ${ }^{*} P<0.05$. The data are representative of at least 3 assessments with 5 different donors.

of inhibitory receptor signaling. Genetic and biochemical studies indicated that high antigen concentration impaired $\mathrm{CD}^{+}$ $\mathrm{T}$ cell polyfunctionality through inhibition of the MAPK/ERK pathway via upregulation of sprouty-2 (SPRY2), a negative regulator of the MAPK/ERK pathway. Based on these findings, we analyzed a cohort of HIV-infected patients and found that SPRY2 is involved in HIV-specific T cell exhaustion. HIV-specific $\mathrm{T}$ cells showed increased levels of SPRY2 mRNA and SPRY2 protein compared with influenza-specific $\mathrm{T}$ cells from the same donors. Furthermore, shRNA-mediated inhibition of SPRY2 enhanced HIV-specific polyfunctionality independently of PD-1 blockade. When shRNA-mediated inhibition of SPRY2 was analyzed in the presence of anti-PD-1, HIV Gag-specific CTL had levels of polyfunctionality similar to the nonexhausted CEF (CMV, EBV, influenza virus) responses. SPRY2 thus appears to mediate inhibition of HIV-specific T cell polyfunctionality independently of the PD-1 pathway. These findings advance our understanding of the molecular control of $\mathrm{T}$ cell polyfunctionality and indicate what we believe to be a novel therapeutic target to reverse $\mathrm{T}$ cell dysfunction associated with chronic viral infection or cancer.

\section{Results}

Antigen concentration regulates polyfunctionality of buman $T$ cells. Autologous moDCs were pulsed with various concentrations of the immunodominant, HLA-A*0201 restricted influenza peptide $\mathrm{M}_{5} 8_{-66}$ and used to stimulate $\mathrm{CD} 8^{+} \mathrm{T}$ cells ex vivo. On day 14 (D14), after 2 rounds of peptide-pulsed moDC stimulation, cell counts and percentage of M1-specific $\mathrm{CD}^{+} \mathrm{T}$ cells were determined. There was a direct correlation between the amount of antigen used, from $10 \mathrm{fM}$ to $10 \mu \mathrm{M}$, to pulse DCs and percentage of M1-specific $\mathrm{CD}^{+} \mathrm{T}$ cells as well as the overall cell number (Figure 1, 
A

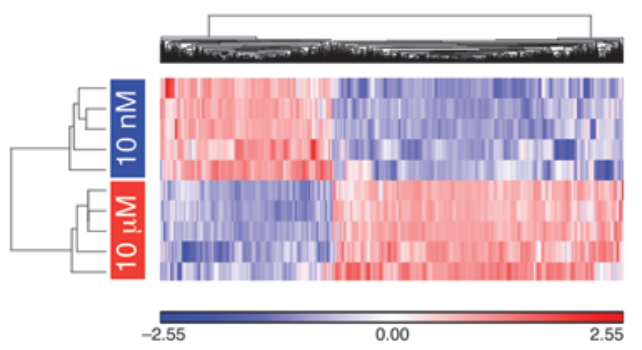

B

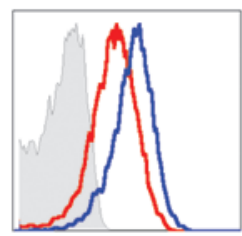

EOMES

C

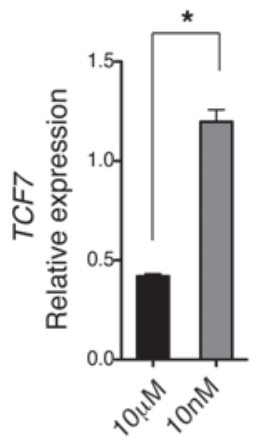

D

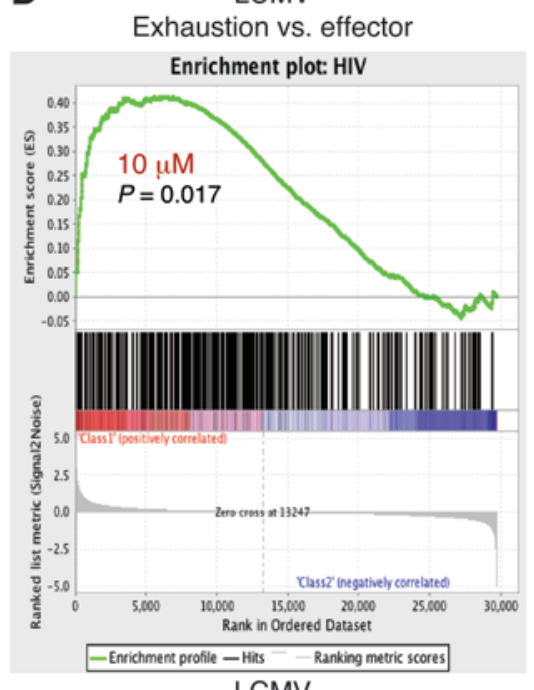

LCMV

Memory vs. effector Enrichment plot: MVSEFF

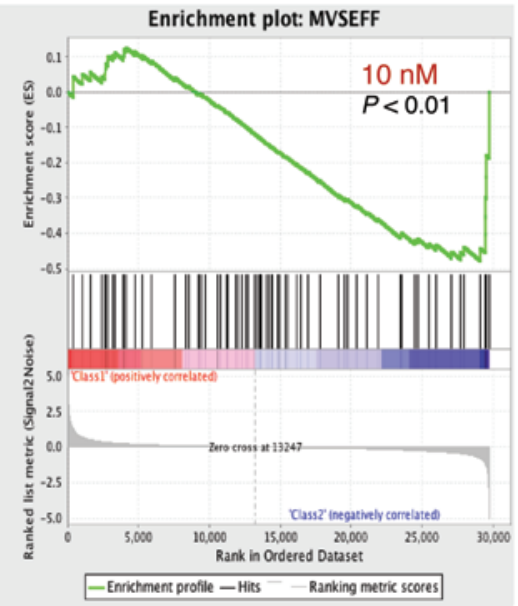

HIV

Progressors vs. controllers Enrichment plot: EXH_V_REST_600

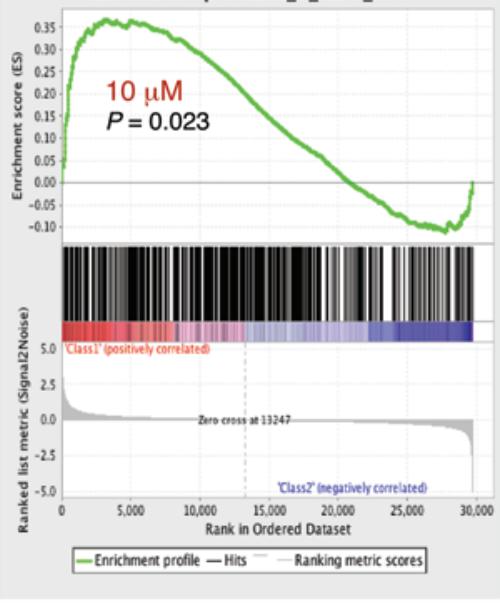

PD-1

Ligation

Enrichment plot: PD-1_JURKAT_001_200

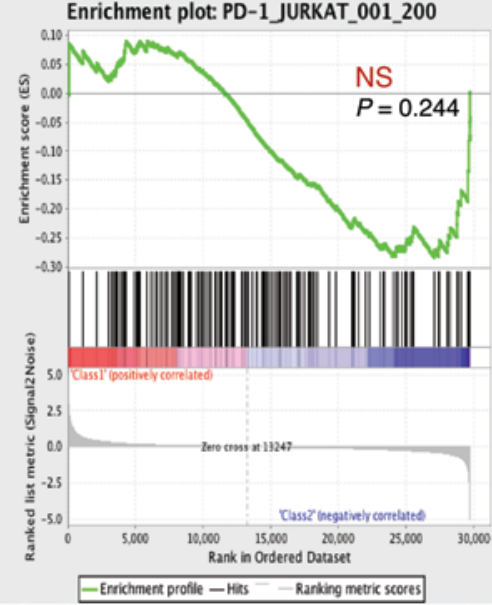

Figure 2

Microarray analysis reveals unique molecular signatures associated with T cell polyfunctionality. (A) Heat map of differentially expressed genes among T cells induced by $10 \mu \mathrm{M}$ moDCs or $10 \mathrm{nM}$ moDCs (adjusted $P<0.05$, fold change $>1.6$ ). Red and blue indicate increased and decreased gene expression, respectively. (B) Expression level of EOMES among T cells induced by 10M moDCs (red) or $10 \mathrm{nM}$ moDCs (blue), determined by flow cytometry. (C) Real-time qPCR validation of TCF7 level. T cells induced with $10 \mu \mathrm{M}$ or $10 \mathrm{nM}$ pulsed moDCs were tetramer sorted and analyzed by qPCR. ${ }^{\star} P<0.05$. (D) GSEA shows that high antigen concentration-induced T cells are enriched with exhaustion signature from LCMV or HIV. In contrast, optimal concentration-induced T cells were enriched with the genetic signature of memory T cells. Molecular signature from PD-1 ligation was not able to distinguish between T cells stimulated with high and optimal antigen concentrations.

A-C). Percentage of M1-specific T cells varied over 40-fold, from approximately $2 \%$ to $85 \%$, and a similar impact was seen on total number of M1-specific CD8 ${ }^{+} \mathrm{T}$ cells.

Antigen concentration also controlled $\mathrm{T}$ cell effector function. On D14, T cells were incubated with M1 peptide-pulsed target cells and analyzed for multiple effector functions. Compared with $\mathrm{T}$ cells induced by $10 \mathrm{nM}$ peptide-pulsed moDCs, T cells induced by high antigen concentration $(10 \mu \mathrm{M})$ had a significantly reduced ability to simultaneously produce multiple cytokines or effector functions (Figure 1, D and E). Only 5.6\% of the M1-specific cells induced with $10 \mu \mathrm{M}$ peptide-pulsed moDCs produced both IL-2 and CD107, and $15.1 \%$ produced both TNF- $\alpha$ and MIP- $1 \beta$. In contrast, when $\mathrm{T}$ cells were induced with $10 \mathrm{nM}$ moDCs, there were significantly larger percentages of $\mathrm{T}$ cells with multiple effector functions. $25.4 \%$ of the M1-specific T cells produced both IL-2 and CD107, and approximately 50\% produced both TNF- $\alpha$ and MIP$1 \beta$. A low antigen concentration, $10 \mathrm{fM}$, also induced a lower percentage of dual-function M1-specific $\mathrm{T}$ cells when compared with the $10 \mathrm{nM}$ antigen concentration, but a higher percentage of dualfunction M1-specific T cells compared with the $10 \mu \mathrm{M}$ antigen. The effector function most sensitive to antigen concentration was IL-2 production, and functions least sensitive were CD107a and MIP-1 $\beta$ (Figure 1E). The loss of IL-2 responses during high antigen concentration stimulation is also observed during chronic infections such as HIV (9), where IL-2 production is the most sensitive and first function lost out of other effector functions. 
A

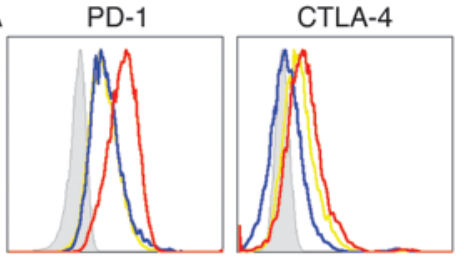

C

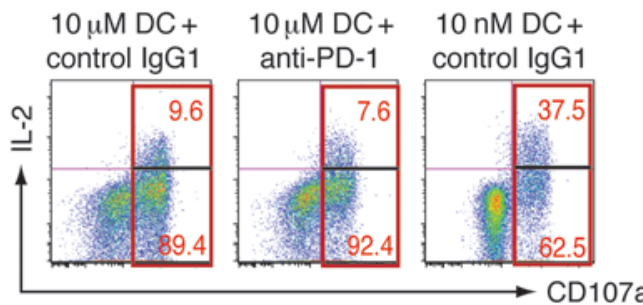

TIM-3
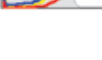

$\operatorname{MDC}+$ control lgG1

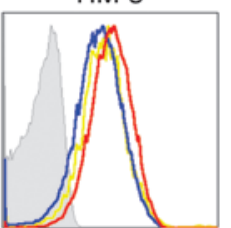

D

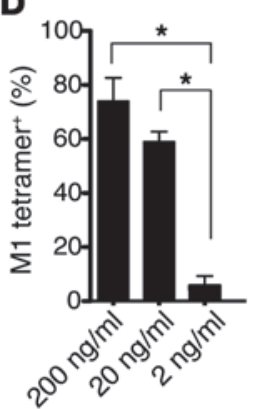

HLA-A2-M1

concentration

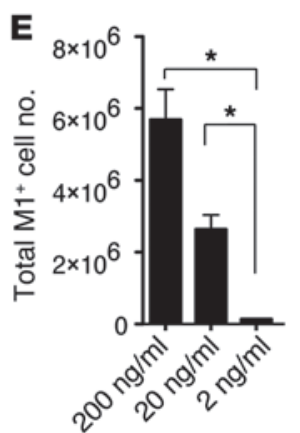

HLA-A2-M1 concentration
B

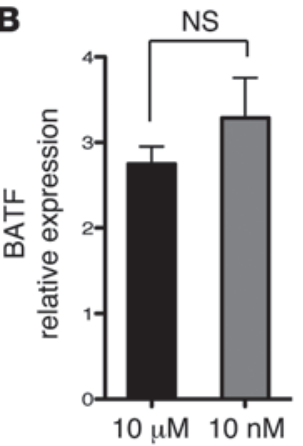

\section{Figure 3}

Polyfunctionality regulation by antigen concentration is independent of inhibitory receptor signaling. (A) On D14, T cells were harvested and stained with anti-CD8 and M1 tetramer, then assayed for inhibitory receptor expression. Representative histograms shown were gated on CD8 ${ }^{+}$, M1 tetramer+ population. Red: $10 \mu \mathrm{M}$. Blue: $10 \mathrm{nM}$. Yellow: $10 \mathrm{fM}$. High antigen concentration-induced M1-specific T cells (10 $\mu \mathrm{M})$ showed higher inhibitory receptor expression except for BTLA. (B) Real-time qPCR analysis of BATF expression level in D14 sorted M1-specific cells. (C) Blockade of PD-1 signaling by anti-PD-1 antibody (clone EH12.2H7) during high antigen concentration stimulation did not improve polyfunctionality. The lack of any effect of anti-PD-1 treatment was seen at the standard dose used of $10 \mu \mathrm{g} / \mathrm{ml}$ (see above) as well as at 10 -fold higher concentrations of anti-PD-1 treatment. Isotype control antibody (clone MG1-45) was added at the same concentration. (D-F) Ex vivo CD8+ T cells stimulated with different concentrations of aAPCs weekly for 2 weeks showed different levels of T cell polyfunctionality. aAPCs were made by conjugating biotinylated HLA-A2-M1 and biotinylated anti-CD28 complex onto anti-biotin microbeads (Miltenyi Biotec). (D) Percentage of tetramer-positive cells and (E) total M1-specific cell expansion. Higher concentrations of aAPCs induced more robust cell expansion, similar to results seen in Figure 1 with peptide-pulsed moDC-induced T cells. (F) Polyfunctionality assessment of aAPC-stimulated M1-specific T cells. High concentration aAPCs induced M1-specific T cells with lower polyfunctionality even in the absence of inhibitory receptors on the aAPCs. The results are representative of 3 independent experiments performed on at least 3 different subjects. ${ }^{*} P<0.05$.

By analyzing overall $\mathrm{T}$ cell polyfunctionality, we determined an antigen concentration that induced the maximal amount of polyfunctional $\mathrm{T}$ cells. When individual $\mathrm{T}$ cell cultures were analyzed for all 5 functions simultaneously, there was a modal distribution for induction of polyfunctional T cells (Figure 1, F and G). $10 \mathrm{nM}$ antigen pulsed moDCs induced the greatest polyfunctional response; $26.4 \%$ of all M1-specific $\mathrm{T}$ cells had all 5 functions (5+ polyfunctional $\mathrm{T}$ cells). This was significantly higher than the response induced by the high antigen concentration, $10 \mu \mathrm{M}, 4.5 \%$ $5+$ polyfunctional M1-specific T cells $(P=0.002)$, or by the low antigen concentration, $10 \mathrm{fM}, 13.7 \% 5+$ polyfunctional M1-specific T cells $(P=0.001)$. There was no further inhibition of $\mathrm{T}$ cell polyfunctionality in response to an even higher antigen concentration, $100 \mu \mathrm{M}$ (data not shown). Within the population of 5+ polyfunctional $\mathrm{T}$ cells, $\mathrm{T}$ cells induced with $10 \mathrm{nM}$ moDCs also produced more IL-2 on a per cell basis (Figure 1H). T cells with the lowest polyfunctionality, induced by $10 \mu \mathrm{M}$ moDCs, were dominated by a population of cells capable of producing MIP- $1 \beta$ and degranulation, but did not produce any cytokines (Supplemental Figure 1; supplemental material available online with this article; doi:10.1172/JCI70510DS1). Upon rechallenge with antigen, T cells induced with $10 \mathrm{nM} \mathrm{M} 1$ antigen-pulsed moDCs also exhibited superior cell expansion (Supplemental Figure 2). Thus, in the following sections of this manuscript, we refer to $10 \mathrm{nM}$ as the optimal antigen concentration and $10 \mu \mathrm{M}$ as the high antigen concentration of peptide used to pulse moDCs.

While these studies analyzed the memory M1-specific $\mathrm{T}$ cell responses, we also analyzed the impact of antigen concentration on naive $\mathrm{CD} 8^{+} \mathrm{T}$ cells. When naive $\mathrm{T}$ cells were stimulated with different concentrations of anti-CD3 and anti-CD28, a simi- 

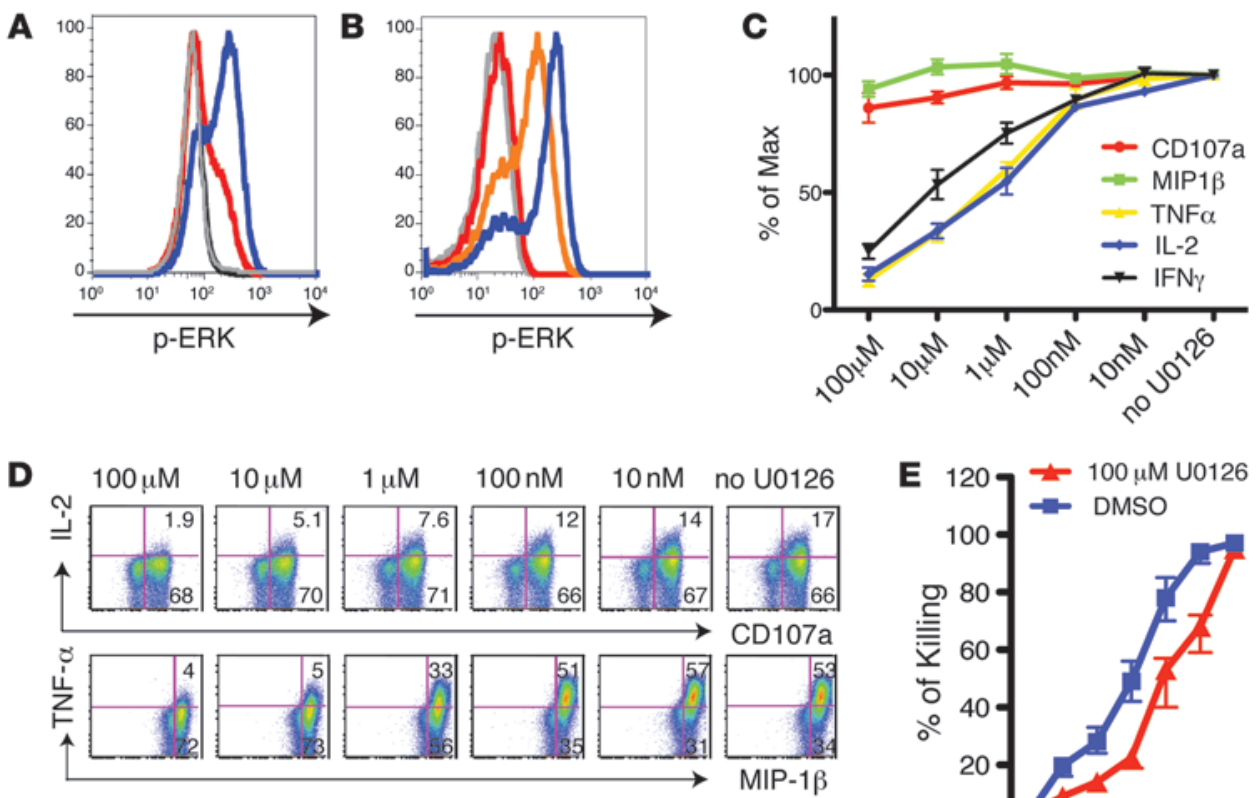

Figure 4

MAPK/ERK pathway controls T cell polyfunctionality. (A) D14 tetramer-sorted high and optimal antigen concentration-stimulated T cells were restimulated with PMA/ionomycin for 10 minutes and analyzed for expression of pERK by flow cytometry. Red, high concentration; blue, optimal concentration; Gray, unstimulated. (B) ERK inhibitor U0126 inhibits pERK expression in a concentration-dependent fashion. T cells stimulated by the optimal concentration of antigen were restimulated with PMA/ionomycin in the presence or absence of varying concentrations of the ERK inhibitor U0126 for 10 minutes. Cells were then stained for pERK expression. Red, $100 \mu \mathrm{M}$ U0126; orange: $1 \mu \mathrm{M}$ U0126; blue, no U0126; gray, unstimulated. (C and D) Optimal concentration-induced T cells were incubated with T2 target cells for 6 hours in the presence of various amounts of U0126 before intracellular cytokine staining and polyfunctionality analysis. U0126 selectively inhibited cytokine secretion but not CD107a upregulation and MIP-1 expression. (E) T cells still mediated effective lysis of peptide-pulsed T2 target cells in the presence of $100 \mu \mathrm{M} U 0126$.

lar modal distribution for induction of polyfunctional $\mathrm{T}$ cells was observed (Supplemental Figure 3). Optimal concentration of anti-CD3/anti-CD28 resulted in $28 \%$ polyfunctional $\mathrm{T}$ cells, while the highest concentration of anti-CD3/anti-CD28 led to only $1.8 \%$ polyfunctional $\mathrm{T}$ cells. Thus, antigen concentration controls $\mathrm{T}$ cell polyfunctionality in both memory and naive $\mathrm{CD} 8^{+}$ $\mathrm{T}$ cell populations.

Regulation of polyfunctionality is independent of the T cell differentiation markers. It is possible that different M1-specific T cell clones are hardwired with different polyfunctionality potential and selectively expanded in response to particular concentrations of antigen. This seemed unlikely as more than $95 \%$ of the M1 tetramerpositive cells were positive for $\mathrm{V} \beta 17 \mathrm{TCR}$, independent of the amount of antigen used for stimulation (data not shown). In addition, it is possible that the $T$ cell differentiation state could also have an impact on their effector functions (19). We characterized $T$ cells stimulated with a range of peptide concentrations using a panel of differentiation markers including CD45RO, CD45RA, CCR7, CD28, and CD27 and found no significant differences (Supplemental Figure 4A) among the populations. T cells from all stimulation conditions also expressed similar levels of bcl-2 and T-bet (Supplemental Figure 4B).

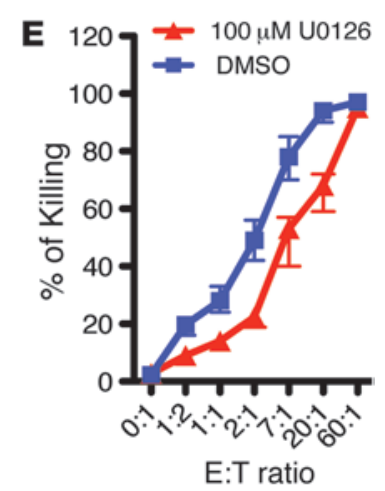

Molecular signature of $T$ cell polyfunctionality. Based on these results, we hypothesized that distinct molecular mechanisms regulate $T$ cell polyfunctionality. To establish the molecular signature of polyfunctionality, antigen-specific $T$ cells were induced by either high or optimal antigen concentration, tetramer sorted on D14, and analyzed for global gene expression. Cut-off point analysis was set at 1.6-fold change and an adjusted $P$ value of less than 0.05 .

628 well-annotated genes were upregulated in high versus optimal antigen concentration-induced $\mathrm{T}$ cells, and 302 genes were downregulated (Figure 2A). Functional grouping performed by the Database for Annotation, Visualization, and Integrated Discovery (DAVID) revealed that most differentially regulated genes are central to lymphocyte function, including inhibitory receptor expression, lymphocyte activation, chemotaxis, cytotoxicity, and antigen presentation (Supplemental Table 1). A full list of differentially expressed genes can be found in Supplemental Table 2.

Compared with high antigen concentration-induced $\mathrm{T}$ cells, highly polyfunctional $\mathrm{T}$ cells induced by the optimal antigen concentration expressed less inhibitory receptors at the mRNA level, including PD-1, 2B4 (CD244), and KLRG1. Microarray data showed that transcription factors that promote $\mathrm{CD}^{+} \mathrm{T}$ cell memory formation, such as EOMES (20) and TCF7 (21), were higher in highly polyfunctional $\mathrm{T}$ cells, and differences were confirmed by flow cytometry or quantitative PCR (qPCR) (Figure 2, B and C). We also found differential expression of multiple chemokines and chemokine receptors, which have also been shown to regulate $\mathrm{T}$ cell differentiation and memory formation $(22,23)$, and a large group of differentially expressed genes were involved in signal transduction.

High antigen concentration-induced T cells are enriched with the molecular signature of $T$ cell exhaustion. GSEA has been used to compare genomic signatures between microarray experiments in order to integrate different phenotypic cellular states (24). GSEA showed that highly polyfunctional $\mathrm{T}$ cells were significantly enriched with the genetic signature for memory T cells (ref. 25 and Figure 2D). In contrast, there was a significant enrichment of the exhausted gene signature (25) among high antigen concentration-induced $\mathrm{T}$ cells, indicating that these $\mathrm{T}$ cells share aspects of global gene expression patterns with $\mathrm{T}$ cells that are exhausted due to chronic lymphocytic choriomeningitis virus (LCMV) infection (Figure 2D). Similar enrichment was found while using the differentially expressed gene list from HIV progressors versus controllers (26). Interestingly, while it has been established that the PD-1/PD-L1 
A
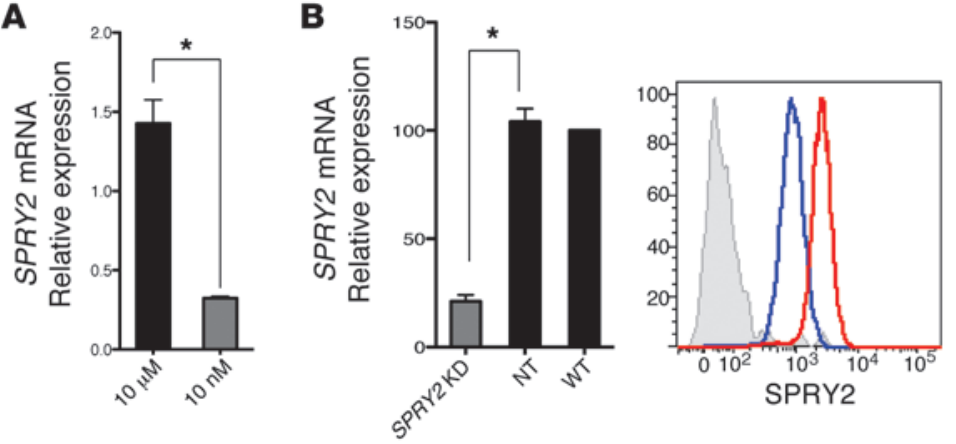

C SPRY2 KD NT
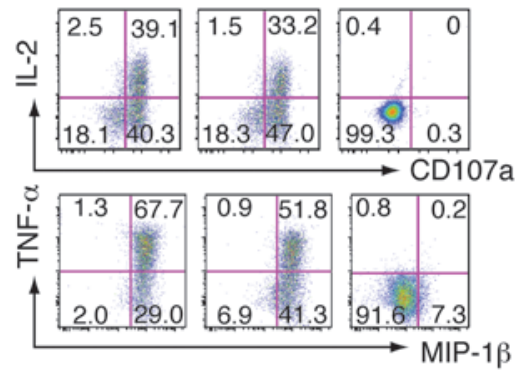

D

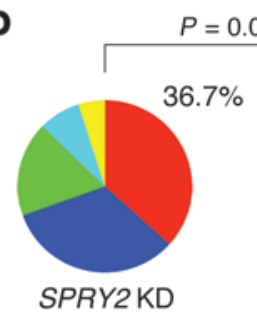

$=0.003$

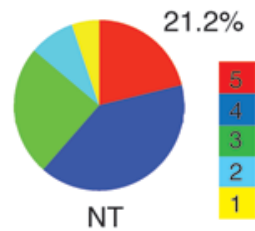

\section{Figure 5}

Upregulation of SPRY2 in high antigen concentration-induced T cells inhibits polyfunctionality. (A) qPCR comparison of SPRY2 expression levels on high and optimal antigen concentration-stimulated $T$ cells. (B) The knockdown efficiency of lentiviral particles containing shRNA targeting SPRY2. Twenty-four hours after the second high antigen concentration moDC stimulation, cells were transduced with either SPRY2 knockdown virus or NT sequence virus. GPCR, flow cytometry staining of SPRY2 (red, NT virus control; blue, SPRY2 knockdown virus; gray, FMO control) and polyfunctionality assessment were performed on D14. (C) Representative flow plots of effector functions in virus-transduced T cells. T cells transduced with SPRY2 knockdown virus exhibit enhanced cytokine production of IL-2 and TNF- $\alpha$ as compared with control NT virus. SPRY2 knockdown had no impact on either CD107a upregulation or MIP-1 $\beta$ production. (D) SPRY2 knockdown virus-transduced T cells showed greater percentage of $5+$ polyfunctional T cells than control virus-transduced T cells. ${ }^{*} P<0.05$. The data are representative of more than 3 independent experiments performed on 3 different subjects. pathway is involved in $\mathrm{T}$ cell exhaustion during chronic viral infections (27), GSEA analysis of the PD-1 ligation signature (26) showed no significant enrichment.

Inhibitory receptor signaling is not required for bigh antigen concentration-induced polyfunctionality inbibition. To specifically study the role of inhibitory receptor signaling in the generation of polyfunctional $\mathrm{T}$ cells, we analyzed the inhibitory receptor expression on $T$ cells induced by different antigen concentrations. Significantly higher levels of multiple inhibitory molecules such as PD-1, CTLA-4, and TIM-3 were seen in M1-specific T cells induced by high versus optimal concentration of antigen (Figure $3 \mathrm{~A}$ ). However, no difference was seen in expression of another inhibitory receptor, BTLA. We took several approaches to analyzing the potential role of signaling through inhibitory receptors, such as PD-1. Expression of basic leucine transcription factor, ATF-like (BATF), an important PD-1 downstream signaling molecule (26), was not upregulated by $\mathrm{qPCR}$, in high antigen concentrationinduced $\mathrm{T}$ cells (Figure 3B). We further analyzed the potential role of PD-1 during high antigen concentration stimulation using anti-PD-1 antibody to block PD-1/PD-L1 interaction $(10 \mu \mathrm{g} / \mathrm{ml}$, clone EH12.2H7). Nevertheless, no increase in polyfunctionality was seen in high antigen concentration-induced $\mathrm{T}$ cells treated with anti-PD-1 (Figure 3C).

In addition to PD-1, there are other inhibitory ligands present on DCs that could potentially modulate polyfunctionality. To analyze the impact of antigen concentration on polyfunctionality in the absence of potential inhibitory molecules, we used artificial APCs (aAPCs), which have no inhibitory receptor ligands. This is a reductionist system in which aAPCs are made by conjugating HLA-A2-Ig-M1 complex (signal 1) and anti-CD28 (signal 2) onto particles $(28,29)$. Higher concentrations of aAPCs with signal 1 plus signal 2 induced significantly more antigen-specific $\mathrm{T}$ cell proliferation (Figure 3, D and E), but lower T cell polyfunctionality (Figure 3F). Similar to moDC stimulation, there was an optimal antigen concentration for aAPC stimulation. Therefore, while signaling through PD-1 and other inhibitory receptors is widely involved in $\mathrm{T}$ cell exhaustion, polyfunctionality regulation due to antigen concentration requires only signal 1 and signal 2 .

$M A P K / E R K$ pathway controls $T$ cell polyfunctionality in response to antigen concentration. To further investigate the molecular mechanism regulating polyfunctionality, we performed GO-BP-FAT analysis on the microarray result and found that the MAPK/ERK pathway is altered in T cells with different levels of polyfunctionality (Supplemental Table 3). The MAPK/ERK pathway is an important signaling pathway that critically regulates a variety of physiological processes, such as cell growth, differentiation, and survival. This pathway also plays important roles in many aspects of lymphocyte biology, such as thymocyte selection, $\mathrm{CD}^{+} \mathrm{T}$ cell differentiation, and $\mathrm{T}$ cell activation (30). However, the role of the MAPK/ERK pathway in T cell polyfunctionality and exhaustion has not been reported.

Differences were seen in the level of phospho-ERK (pERK) upregulation in $\mathrm{T}$ cells induced by either high or optimal antigen concentration. $67 \%$ of the optimal antigen concentration-induced T cells upregulated pERK in response to stimulation, while only $33 \%$ of the poorly polyfunctional $\mathrm{T}$ cells showed increased levels of pERK (Figure 4A). Upregulation of pERK was shown to be specific, as treatment of cells with U0126, a MEK1/2 specific inhibitor, inhibited ERK phosphorylation in a concentration-dependent fashion, with complete inhibition of pERK seen with $100 \mu \mathrm{M}$ U0126 (Figure 4B).

If the MAPK/ERK pathway differentially controls effector functions involved in polyfunctionality, such as cytokine production, one should be able to convert $5+$ polyfunctional antigen-specific $T$ cells to low polyfunctional $T$ cells by inhibiting ERK phosphorylation. In the presence of U0126, there was a dose-dependent inhibition of cytokine production (IL-2, TNF, and IFN- $\gamma$ ), with little effect on CD107 and MIP-1 $\beta$ expression in optimal antigen concentration-induced $\mathrm{T}$ cells. There was an approximately 10 -fold reduction in the amount of IL- 2 and 
A

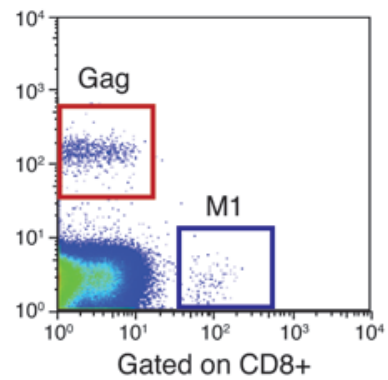

C

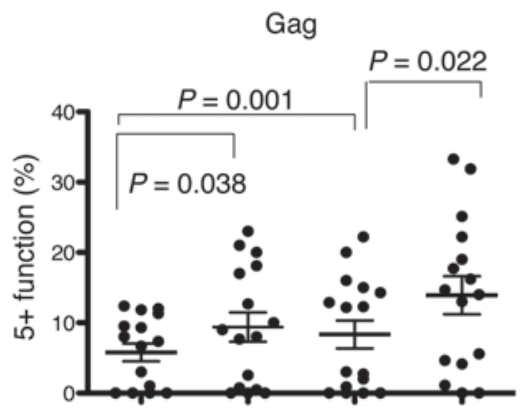

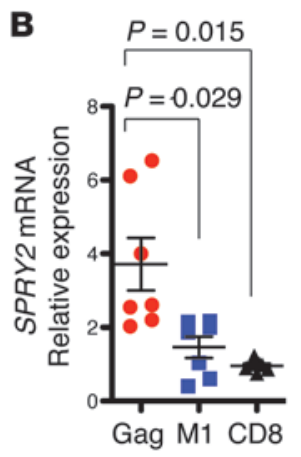
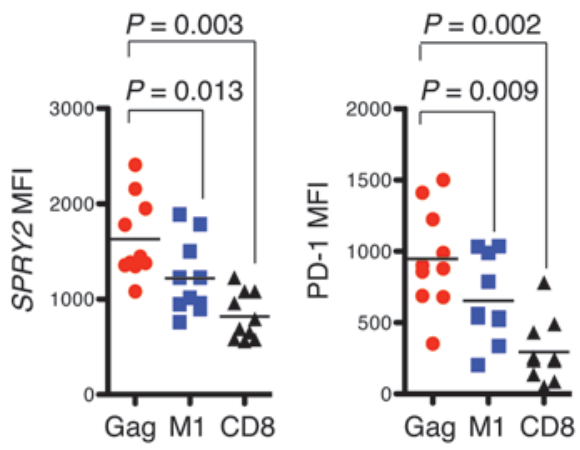

D $\mathrm{Nef}$
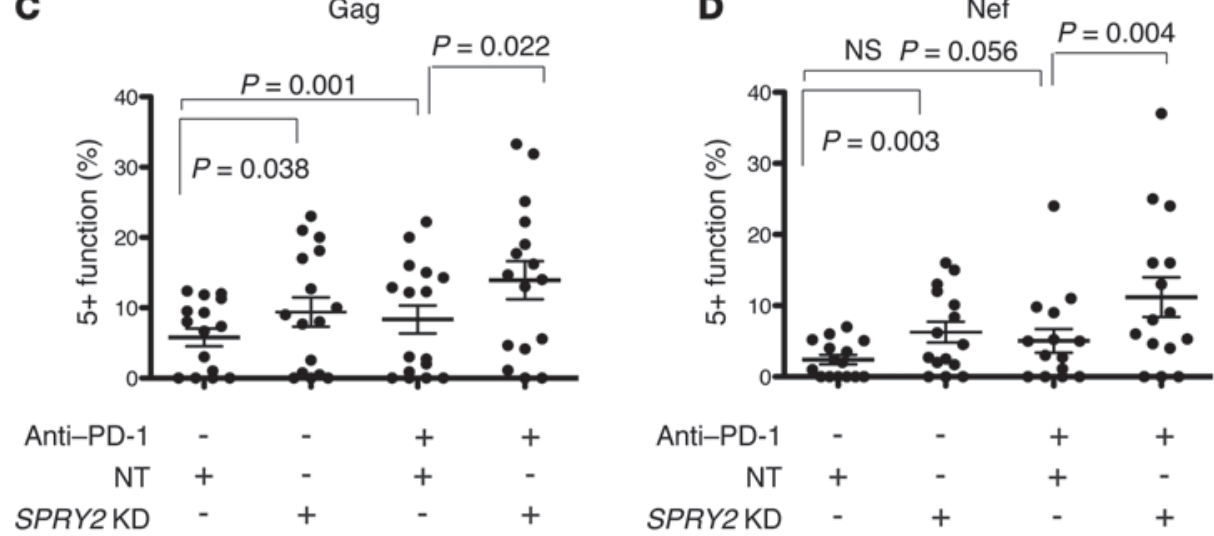

E

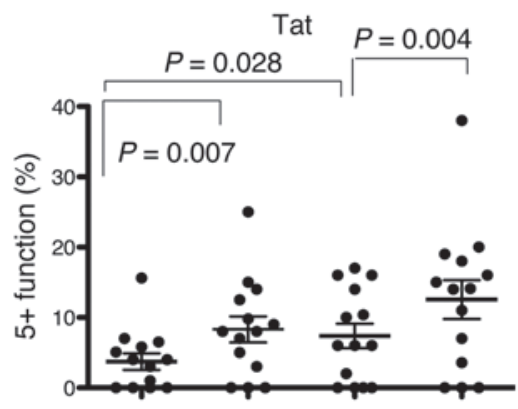

$\begin{array}{rcccc}\text { Anti-PD-1 } & - & - & + & + \\ \text { NT } & + & - & + & - \\ \text { SPRY2 KD } & - & + & - & +\end{array}$

$\mathbf{F}$

CEF

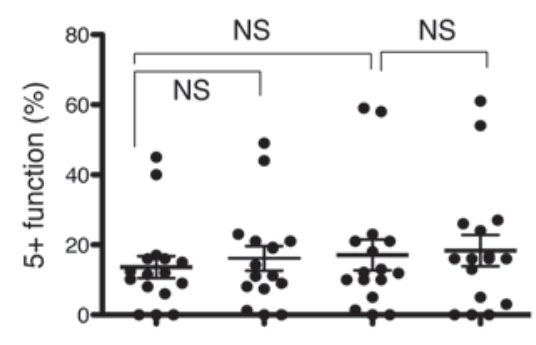

$\begin{array}{rcccc}\text { Anti-PD-1 } & - & - & + & + \\ \text { NT } & + & - & + & - \\ \text { SPRY2 KD } & - & + & - & +\end{array}$

\section{Figure 6}

SPRY2 inhibition enhances HIV-specific T cell polyfunctionality. (A and B) SPRY2 expression was studied in 12 HIV-infected HLA-A2 ${ }^{+}$patients. HIV-Gag-specific T cells and influenza M1-specific T cells were sorted by pentamer/tetramer staining and mRNA extracted from Gag- and M1-specific cells for qPCR analysis. (A) An example of flow cytometry-based simultaneous analysis of Gag- and M1-specific T cells from PBMCs of an HIV-infected donor. (B) qPCR and flow cytometry analysis of SPRY2 and PD-1 expression in Gag-specific, M1-specific, and total CD8 ${ }^{+} \mathrm{T}$ cells from HLA-A2+ HIV donors. Statistical analyses were performed by nonparametric Wilcoxon matched-pairs signed rank test. (C-F) PBMCs from 19 HIV-infected patients were activated with soluble anti-CD3, anti-CD28, and a mix of CEF/HIV peptide pools. Some cultures were also treated with anti-PD-1 $(10 \mu \mathrm{g} / \mathrm{ml})$ to block PD-1 signaling during activation. 24 hours later, cells were transduced with SPRY2 knockdown (KD) or NT control lentivirus. On D7 after virus transduction, PBMCs were stimulated with CEF or HIV Gag, Nef, and Tat peptide pools for 6 hours and analyzed for polyfunctionality. (C-E) Inhibition of SPRY2 expression led to augmented HIV-specific T cell polyfunctionality above and beyond that seen by inhibition of PD-1 pathway alone. (F) PD-1 blockade alone or in combination with SPRY2 inhibition had no significant effect on the CEF-specific response.

TNF- $\alpha$ made by M1-specific CD8 ${ }^{+} \mathrm{T}$ cells (Figure 4, C and D). However, $\mathrm{CD}^{+} \mathrm{T}$ cell-mediated lysis of target cells was largely insensitive to U0126 treatment (Figure 4E). Overall, the data indicate that cytokine secretion and polyfunctionality but not lytic ability or MIP- $1 \beta$ expression is dependent on the MAPK/ ERK pathway. Thus, the differential consequences of optimal and high antigen concentrations could be replicated by biochemical inhibition of the MAPK/ERK pathway. 
Upregulation of SPRY2 in high antigen concentration-induced T cells inhibits polyfunctionality. One gene identified in the microarray gene list (Supplemental Table 1) known to affect the MAPK/ERK pathway is SPRY2. Sprouty proteins are a well-conserved family known to mediate the negative feedback regulation of the MAPK pathway $(31,32)$. Sprouty proteins bind to Grb2 and other components of the MAPK pathway, preventing the upregulation of $\operatorname{PERK}(33,34)$.

qPCR analysis confirmed that SPRY2 is upregulated in high antigen concentration-induced $\mathrm{CD}^{+} \mathrm{T}$ cells (Figure $5 \mathrm{~A}$ ) and upregulation of SPRY2 is downstream of ERK activation, as it is inhibited by U0126 treatment added during high antigen concentration stimulation (Supplemental Figure 5). To determine whether SPRY2 mediates suppression of polyfunctionality in high antigen concentration-induced $T$ cells, we engineered lentiviral particles containing shRNA-targeting SPRY2 in order to inhibit its expression. SPRY2 knockdown virus efficiently inhibited mRNA expression of SPRY2, by approximately $80 \%$, while control nontarget (NT) virus had no effect (Figure 5B). SPRY2 knockdown also led to a significant decrease in SPRY2 protein levels as determined by flow cytometry (Figure 5B). The MFI of SPRY2 in the SPRY2 knockdown cells decreased from 1860 to 910 . Upon antigen stimulation, the SPRY2 knockdown T cells produced more cytokines than the control lentivirus-infected T cells (Figure 5C), and overall, the percentage of $5+$ polyfunctional $\mathrm{T}$ cells increased from approximately $21 \%$ to $37 \%$ (Figure 5D). Thus, high antigen concentration stimulation leads to lower $\mathrm{CD}^{+} \mathrm{T}$ cell polyfunctionality through upregulation of $S P R Y 2$, which inhibits the MAPK/ERK pathway.

SPRY2 controls HIV-specific T cell polyfunctionality. As compared with acute infection such as influenza, HIV-specific CTL responses in infected patients are known to have significantly lower levels of polyfunctionality, and CTL dysfunction is not restored by antiretroviral therapy $(9,35)$. Recent studies indicate that inhibition of the PD-1 pathway partially reverses low polyfunctionality associated with chronic viral infection (13). However, the effects of antiPD-1 treatment were modest $(14,15)$ and indicated that additional mechanisms control HIV-specific T cell polyfunctionality.

We hypothesized that HIV-specific T cells also upregulate $S P R Y 2$ and that upregulation of SPRY2 is responsible for the low levels of T cell polyfunctionality seen in HIV-specific responses. PBMCs from 7 HLA-A2-positive HIV-infected patients were sorted on the basis of pentamer/tetramer staining into either HIV Gag- or influenza M1-specific CTL (Figure 6A). Within CD8 ${ }^{+}$ $\mathrm{T}$ cells, the average percentage of Gag-specific T cells was $0.45 \%$ as compared with $0.04 \%$ of M1-specific T cells $(P=0.03$, data not shown). qRT-PCR on these populations showed that SPRY2 was upregulated in the Gag-specific T cells compared with both the $\mathrm{M}-1$-specific T cells $(P=0.029)$ and nonspecific $\mathrm{CD}^{+} \mathrm{T}$ cells $(P=0.015)$ from the same donors. Flow cytometry staining for SPRY2 showed that Gag-specific T cells express higher levels of SPRY2 protein and PD-1 (Figure 6B). Consistent with previous findings (9), these cells are less polyfunctional when analyzed directly ex vivo (Supplemental Figure 6).

We next studied the effect of SPRY2 knockdown on HIV Gag-, Nef-, and Tat-specific CTL responses (Figure 6, C-F). Inhibition of $S P R Y 2$ substantially enhanced all the HIV-specific polyfunctional responses independently of PD-1 blockade. In addition, inhibition of SPRY2 in combination with anti-PD-1 treatment further augmented polyfunctional responses (Figure 6, C-F). By combining anti-PD-1 with SPRY2 inhibition, HIV Gag-specific polyfunctionality improved to levels seen in the CEF-specific (CMV, EBV, and influenza) responses. In contrast, neither SPRY2 inhibition nor PD-1 blockade had a significant effect on CEF-specific responses from the same donors (Figure $6 \mathrm{~F}$ ). These findings indicate that $S P R Y 2$ upregulation inhibited HIV-specific T cell polyfunctionality independently of the PD-1 pathway.

\section{Discussion}

Polyfunctionality has emerged as a highly significant predictor of protective immunity $(1,2,7,9,18)$. In this report, we generated antigen concentration-dependent influenza-specific CD8 ${ }^{+}$ $T$ cells with differing levels of polyfunctionality. An optimal concentration of antigen, resulting in highly polyfunctional $\mathrm{T}$ cells, was determined, and microarray analysis showed that these $T$ cells were enriched for a memory $\mathrm{T}$ cell signature. In contrast, high antigen concentration-induced $T$ cells that were markedly less polyfunctional were enriched for a $\mathrm{T}$ cell exhaustion signature. These poorly polyfunctional $\mathrm{T}$ cells had high levels of inhibitory receptor expression, but inhibitory receptor signaling did not regulate polyfunctionality in this system. By utilizing the aAPC system, we demonstrated that high antigen concentration alone could regulate polyfunctionality. Through these findings, we identified the MAPK/ERK pathway and SPRY2 as regulators of polyfunctionality in high antigen concentration stimulation and validated the importance of SPRY2 in chronic HIV infection.

The molecular profile of highly polyfunctional $\mathrm{T}$ cells is enriched with memory signature genes and is consistent with the idea that the polyfunctional state is associated with memory T cell development $(1,19,36)$. Two key genes identified as part of the polyfunctional signature were EOMES and TCF7, both known to be indispensable for optimal $\mathrm{CD}^{+} \mathrm{T}$ cell memory formation $(20,37)$. The mechanisms governing their expression levels are not entirely clear, but here we identified antigen concentration as an important factor. The finding that optimal antigen concentration induced higher levels of TCF7 and EOMES could identify the molecular mechanism linking optimal antigen concentration to superior $\mathrm{T}$ cell memory formation.

High antigen concentration induced the most robust proliferation, but the lowest levels of $\mathrm{T}$ cell polyfunctionality. The dominant subpopulation of high antigen concentration-induced T cells did not produce any cytokines, but these $T$ cells efficiently mediated target cell recognition and killing. Thus, high antigen concentration-induced $T$ cells were not anergized. These $T$ cells, which exhibit poor cytokine production, low proliferative potential, and high levels of KLRG1, may represent short-lived effector $T$ cells driven toward terminal differentiation during antigenic stimulation, albeit they did not yet exhibit the CD28- and CD27phenotypes (Supplemental Figure 4).

Chronic viral infection is also associated with high antigen load, $\mathrm{T}$ cell exhaustion, and the loss of polyfunctionality. The loss of each individual function in our system follows the hierarchical order for loss of functions observed in HIV and LCMV infections (8). In brief, IL-2, a homeostatic cytokine that promotes $\mathrm{T}$ cell proliferation, is lost first. Subsequently, secretion of effector cytokines, such as TNF- $\alpha$ and IFN- $\gamma$, is impaired. In contrast, upregulation of CD107a and MIP-1 $\beta$ are least affected (9). Enrichment of the T cell exhaustion signature in the high antigen concentration-induced $T$ cells indicates that these $T$ cells share certain biological pathways with exhausted T cells seen in HIV (26) and chronic LCMV (25) infection. Thus, high antigen concentration-induced $\mathrm{T}$ cells may represent cells "on their way" to becoming completely exhausted. 
Inhibitory receptor upregulation is a key feature of T cell exhaustion (10) and often characterized by active suppression mediated through the PD-1/PD-L1 pathway $(13,14)$. Although the poorly polyfunctional $\mathrm{T}$ cells expressed more inhibitory receptors, the poorly polyfunctional phenotype was independent of inhibitory receptor signaling. This is evident by the fact that $\mathrm{PD}-1 / \mathrm{PD}-\mathrm{L} 1$ blockade did not attenuate high antigen concentration-induced loss of polyfunctionality and that the effect of high antigen concentration was seen even when using a signal $1 / 2$ only-aAPC system to induce poorly polyfunctional T cells. Additional evidence comes from the lack of enrichment of the PD-1 molecular signature, such as upregulation of BATF, in the high antigen concentration-induced T cells. Overall, our data show that TCR signaling strength alone determines $T$ cell polyfunctionality.

Our study identified SPRY2 as a regulator of T cell polyfunctionality and showed that SPRY2 expression level is dependent on antigen concentration. Sprouty proteins were originally identified in Drosophila as regulators for embryonic development, and mutations in sprouty proteins were found in various human cancers (31). All sprouty proteins were found to be negative regulators of growth factor signaling, especially the MAPK/ERK pathway (32, 33). A potential role of sprouty proteins has been seen in $\mathrm{T}$ cell activation where both SPRY1 and SPRY2 inhibit T cell activation and IL-2 production $(34,38)$. SPRY1-deficient mice have been shown to have enhanced antitumor $\mathrm{T}$ cell responses (39). Interestingly, a recent microarray analysis indicated that $S P R Y 2$ is upregulated in $\mathrm{CD} 8^{+} \mathrm{CXCR} 1^{-} \mathrm{CD} 27^{-} \mathrm{CD} 28^{-} \mathrm{T}$ cells capable of producing IL-2 (40). However, the physiological role of SPRY2 was not validated in these studies. Given the importance of the MAPK pathway in immune system signaling, it is likely that sprouty proteins could also regulate the outcome of diverse immune responses. During immune response against infection, upregulation of sprouty proteins might be beneficial to the host as a strategy for preventing immune system overactivation and subsequent immunopathology. On the other hand, modulation of SPRY2 expression could be a potential way to enhance $T$ cell polyfunctionality and immunological memory during chronic infections.

Authors have previously reported trying to improve HIV- or $\mathrm{HCV}$-specific polyfunctional $\mathrm{T}$ cell responses by blocking inhibitory receptor-ligand interactions, such as PD-1/PD-L1 (13-15, 41). To date, the improvement in $\mathrm{T}$ cell function has been modest and probably is limited only to less terminally differentiated cells (42). Here, we show that inhibition of SPRY2 expression was associated with improvement of HIV-specific polyfunctional responses above and beyond the effects of PD-1 blockade. As a result, SPRY2 could be a novel therapeutic target for reversing $\mathrm{T}$ cell exhaustion to be used in combination with inhibitory receptor blockade. The finding that CEF-specific T cells, which remain highly polyfunctional in HIV patients, did not respond to SPRY2 inhibition indicates that the expression level of SPRY2 could be related to different $\mathrm{T}$ cell outcomes of viral infections and additional studies on the regulatory mechanisms of its expression are warranted.

Our study mechanistically reveals the need to choose optimal antigen doses for immunization to achieve effective immunological memory and protection. While it is hard to extrapolate from the doses of antigen used in our in vitro studies to specific vaccine doses, simply increasing the dose of antigen could result in generation of poorly polyfunctional $\mathrm{T}$ cells with less memory potential and might not be an appropriate approach for at-risk populations who exhibit inferior responses to standard vaccinations. Thus, the human $\mathrm{T}$ cell polyfunctionality signature and the molecular mechanisms involving SPRY2 and the MAPK/ERK pathway, as identified in the current study, can have important clinical applications such as identifying desirable immune responses in response to immunization and infection.

\section{Methods}

Study subjects and purification of primary human PBMCs and $C D 14^{+}$and $C D 8^{+}$ $T$ cells. PBMCs from 5 healthy HLA*0201-positive donors and 15 HIVinfected patients were isolated by Ficoll-Paque PLUS gradient centrifugation following the manufacturer's protocol (GE Healthcare). The HIV-infected, HAART-treated aviremic patients were recruited from Johns Hopkins and Case Western Reserve University. The average age of donors was 49 years old. For some experiments, $\mathrm{CD} 14^{+}$and $\mathrm{CD}^{+} \mathrm{T}$ cells were further purified from fresh PBMCs using the $\mathrm{CD} 14^{+}$cell-positive selection and $\mathrm{CD} 8^{+} \mathrm{T}$ cell-negative selection kits according to the manufacturer's instructions (Miltenyi Biotec).

Generation of peptide-loaded moDCs and aAPCs. The generation of moDCs was done by following the standard protocol as previously described (28, 43). Briefly, $\mathrm{CD} 14^{+}$cells were cultured in complete RPMI supplemented with $5 \%$ autologous plasma, $100 \mathrm{ng} / \mathrm{ml}$ human granulocyte-macrophage colonystimulating factor, and $50 \mathrm{ng} / \mathrm{ml} \mathrm{IL}-4$. After 6 days of culture, a maturation cocktail containing $10 \mathrm{ng} / \mathrm{ml}$ TNF- $\alpha, 10 \mathrm{ng} / \mathrm{ml} \mathrm{IL-1,} 1000 \mathrm{U} / \mathrm{ml}$ IL- 6 and $1 \mu \mathrm{g} / \mathrm{ml}$ prostaglandin E2 was added to culture. MoDCs were harvested on D7, and the mature DC phenotype was confirmed by flow cytometric analysis of CD80, CD86, and HLA-DR. moDCs were then pulsed with influenza virus matrix peptide M1 (58-66; GILGFVFTL) at different concentrations in serum-free media for an hour at $37^{\circ} \mathrm{C}$. Peptide-pulsed moDCs were then washed extensively to remove free peptide in solution before use.

For generation of aAPCs, M1 peptide-loaded HLA-A2-Ig and anti-CD28 (clone 9.3) were biotinylated and coupled to anti-biotin coated microbeads (Miltenyi Biotec) and stored at $4^{\circ} \mathrm{C}(29)$ before use.

In vitro expansion of $M 1$-specific $C D 8^{+} T$ cells. Three million freshly isolated $\mathrm{CD}^{+} \mathrm{T}$ cells were cocultured with 1 million moDCs in complete RPMI medium containing $5 \%$ autologous plasma, $3 \% \mathrm{~T}$ cell growth factor (28), and antibiotics. For micro-aAPC stimulation, different amounts of microaAPCs were cocultured with CD8 ${ }^{+} \mathrm{T}$ cells. On D7 and weekly thereafter, $\mathrm{T}$ cells were harvested, counted, and replated at the same T cell/APC density. Influenza M1-antigen specificity was determined by using HLA-M1specific A*0201 PE or APC tetramers (GILGFVFTL; Beckman Coulter).

Detection of intracellular cytokines to assess polyfunctionality. For experiments involving M1-specific T cells, $2 \times 10^{5} \mathrm{CD}^{+} \mathrm{T}$ cells were incubated with $1 \times 10^{5}$ target cells ( $\mathrm{HLA}^{*} 0201$-positive $\mathrm{T} 2$ cell line) at $37^{\circ} \mathrm{C}$ in the presence of monensin, brefeldin A and anti-CD28/CD49d costimulation (all from BD Biosciences). Anti-CD107a was added at the start of stimulation. Target cells were pulsed with $\mathrm{M} 1$ peptide at a concentration of $1 \mu \mathrm{M}$ for an hour and washed twice before use. Unpulsed target cells were used as background stimulation. After 6 hours, cells were washed twice with FACS wash buffer and then stained with viability dye, anti-CD3, and anti-CD8 for 20 minutes. Cells were then fixed and permeabilized with the Cytofix/Cytoperm kit (BD Biosciences) following the manufacturer's protocol. A cocktail of fluorophore-conjugated antibodies containing anti-IL-2, anti-TNF- $\alpha$, anti-IFN- $\gamma$, and anti-MIP-1 $\beta$ was added to the cells and stained for an hour. For experiments involving HIV-specific T cell responses, $2 \times 10^{5}$ PBMCs were stimulated with different peptide pools at a concentration of $0.5 \mu \mathrm{g} / \mathrm{ml}$ per peptide (Gag, Nef, Tat peptide pools, NIH AIDS reagent program; CEF peptide pool, Anaspec) for 6 hours for effector function detection.

Complete combinations of different effector function were performed using the Boolean gate platform of FlowJo version 9.3.1 software (TreeStar). Reported data were further adjusted by background subtraction. Polyfunctionality pie charts were generated by SPICE (Data Mining 
and Visualization Software for Multicolor Flow Cytometry) version 5.2. (National Institute of Allergy and Infectious Diseases [NIAID], NIH) (44).

Microarray experiment, data analysis, and $q P C R$ validation. More than half a million M1-specific T cells induced by $10 \mu \mathrm{M}$ or $10 \mathrm{nM}$ moDCs from 5 experiments on 3 donors were tetramer sorted and preserved in TRI reagent (Molecular Research Center) at $-80^{\circ} \mathrm{C}$. RNA extraction and microarray experiments were performed by Miltenyi Biotec. Hybridization was performed with single-colored Whole Human Genome $8 \times 60 \mathrm{~K}$ Oligo Microarrays (Agilent Technologies). Gene expression data were analyzed using Partek Genomic Suite. Each sample was normalized and $\log _{2}$ transformed before being compared by $t$ test $(P<0.05)$. Genes with fold changes of more than 1.6 were selected for further analysis. Differential expressed genes were functionally grouped and annotated using DAVID Bioinformatics Resources 6.7 (NIAID, NIH; available at http://david.abcc.ncifcrf. gov/home.jsp) (45). Functional annotation and pathway identification were performed using the Gene Ontology (GO) fat category, as provided by the DAVID platform. Validation of significant changes $(P<0.05)$ in the expression of candidate genes were done by real-time qPCR (TaqMan gene expression assay) using ACTB or HPRT1 as internal controls. The complete microarray data set is available from GEO (GSE39761).

GSEA. GSEA (46) was used to identify gene sets that are significantly enriched in either high concentration $(10 \mu \mathrm{M})$ or optimal concentration $(10 \mathrm{nM})$ stimulation. The input to GSEA is the $\log _{2}$ transformed expression ratio for each gene. In the scenario in which 1 gene is measured by multiple probes, we used the maximum expression ratio of all probes for that gene. GSEA (46) calculates a running sum of the statistic for each gene set. The test statistic is the maximum of the running sum (enrichment score), and permutation tests are performed to evaluate the empirical $P$ value of the enrichment. Gene sets for LCMV exhaustion versus normal effector and memory T cells (25) were provided by John Wherry (University of Pennsylvania School of Medicine, Philadelphia, Pennsylvania, USA). Gene sets for HIV progressor and PD-1 signaling (26) were provided by Nicolas Haining (Dana-Farber Cancer Institute, Boston, Massachusetts, USA).

pERK staining and cytotoxicity assay. pERK detection was based on protocols as previously described (47). Briefly, M1-specific T cells were stimulated with PMA/ionomycin for 10 minutes before fixation with $2 \%$ formaldehyde and permeabilized with $90 \%$ methanol. Staining was performed with anti-phospho-p44/42 MAPK (ERK1/2) antibody from Cell Signaling (clone E10). Flow cytometry-based cytotoxicity assay (48) was used to investigate the effect of ERK inhibition on T cell-mediated HLAA2-positive T2 cell killing.

Production of shRNA-containing lentiviral particles and T cell transduction. shRNA sequence (5'-CTGAACAGAGACTGCTAGGATCATCCTTC-3') targeting the human SPRY2 gene was cloned into pLKO.1 puro plasmid (provided by Joel Pomerantz, Johns Hopkins University School of Medicine). Viral particles were made by cotransfecting 20 ng of the PLKO plasmid plus $15 \mathrm{ng}$ delta 8.9 plasmid and $10 \mathrm{ng}$ pCMV-VSV-G into HEK293T cells (plated 24 hours earlier at $2.5 \times 10^{6}$ cells/dish) via calcium phosphate precipitation as previously described (49). Transduction of primary M1-specific T cells was performed 24 hours after DC stimulation by spinning at $300 \mathrm{~g}$ for 90 minutes in the presence of $8 \mu \mathrm{g} / \mathrm{ml}$ polybrene (Sigma-Aldrich). 24 hours after transduction, culture medium containing $1.5 \mu \mathrm{g} / \mathrm{ml}$ puromycin was used to select for virus-transduced T cells.
RNA isolation (Cells-to-CT kit; Invitrogen) and qPCR (TaqMan gene expression assay) for knockdown efficiency and polyfunctionality analysis were performed 6 days after viral transduction. Similar transduction procedures were performed with HIV-specific patients' PBMCs. PBMCs were thawed and rested overnight before activation with soluble anti-CD3 (clone HIT3a, $1 \mu \mathrm{g} / \mathrm{ml}$; Biolegend) and anti-CD28 (1 $\mu \mathrm{g} / \mathrm{ml}$, clone CD28.2; Biolegend) in the presence of Gag/Nef/Tat/CEF peptide pools and transduced with lentivirus. Anti-PD-1 antibody $(10 \mu \mathrm{g} / \mathrm{ml}$, clone EH12.2H7; Biolegend) was used to block PD-1 signaling. Isotype control antibody (clone MG1-45; Biolegend) was added to control cultures.

HIV-specific T cell sorting and measurement of SPRY2 expression. HIV Gagantigen specificity was determined by using HLA-A2 restricted PE pentamer (SLYNTVATL; Proimmune). CD8 ${ }^{+} \mathrm{T}$ cells were purified from 7 HLA-A2 ${ }^{+}$ HIV patients from the study subjects and stained with HIV-Gag-pentamer (PE) and M1-tetramer (APC). For each donor, more than 1000 antigenspecific $\mathrm{T}$ cells were sorted for mRNA extraction and CDNA conversion using the Cells-to-Ct kit (Invitrogen). After preamplification using the TaqMan PreAmp Mastermix Kit (Invitrogen), qPCR was performed using TaqMan gene expression assays and SPRY2 expression level were adjusted to HPRT1 expression.

For some donors, unsorted PBMCs were stained with Gag-specific pentamer or M1-specific tetramers, anti-PD-1, and anti-CD8 for an hour. Cells were fixed, permeabilized, and stained with APC-conjugated anti-human SPRY2 antibody (clone ab60719; Abcam) for an additional 2 hours. Cells were then washed twice before acquisition by flow cytometry.

Statistics. Statistical comparisons between different polyfunctionality pie charts were performed by permutation test set at 10,000 repetitions using SPICE. Other statistical analyses were performed by Prism version 5 (GraphPad). For comparison between 2 groups of related samples, the nonparametric Wilcoxon matched-pairs signed rank test was used. The Mann-Whitney $U$ test was used to detect the differences between 2 independent groups of samples. A $P$ value of less than 0.05 was considered statistically significant.

Study approval. This study was approved by the Johns Hopkins Institutional Review Board and the University Hospital Institutional Review Board (Cleveland, Ohio, USA). Informed consent was obtained from all subjects.

\section{Acknowledgments}

This work was supported by grants NCI RO1 CA 108835 and NIAID PO1 AI072677 (to J.P. Schneck); AI080313, AI36219, and VA CSR\&D Merit (to D.H. Canaday); and grants from the Bill and Melinda Gates Foundation (to D.E. Griffin). The authors thank Wen-Hsuan Lin and Rupak Shivakoti for their assistance with the multicolor flow cytometry. We also thank Michael Edidin and Joan Glick Bieler for critical review of this manuscript.

Received for publication April 16, 2013, and accepted in revised form September 27, 2013.

Address correspondence to: Jonathan P. Schneck, Department of Pathology and Medicine, Johns Hopkins University School of Medicine, Miller Research Building, 733 N Broadway, Rm 639, Baltimore, Maryland 21205, USA. Phone: 410.614.4589; Fax: 443.287.0993; E-mail: jschneck1@jhmi.edu.

\footnotetext{
1. Seder RA, Darrah PA, Roederer M. T-cell quality in memory and protection: implications for vaccine design. Nat Rev Immunol. 2008;8(4):247-258.

2. Almeida JR, et al. Superior control of HIV-1 replication by CD8 $+\mathrm{T}$ cells is reflected by their avidity, polyfunctionality, and clonal turnover. J Exp Med. 2007;204(10):2473-2485.

3. Rodrigue-Gervais IG, et al. Dendritic cell inhibi-
}

tion is connected to exhaustion of CD8 $+\mathrm{T}$ cell polyfunctionality during chronic hepatitis $\mathrm{C}$ virus infection. JImmunol. 2010;184(6):3134-3144.

4. Aagaard $\mathrm{C}$, et al. Protection and polyfunctional $\mathrm{T}$ cells induced by Ag85B-TB10.4/IC31 against Mycobacterium tuberculosis is highly dependent on the antigen dose. PLoS One. 2009;4(6):e5930. 5 . Ahmadzadeh $\mathrm{M}$, et al. Tumor antigen-specific
CD8 T cells infiltrating the tumor express high levels of PD-1 and are functionally impaired. Blood. 2009;114(8):1537-1544

6. Baitsch L, et al. Exhaustion of tumor-specific CD8 $\mathrm{T}$ cells in metastases from melanoma patients. J Clin Invest. 2011;121(6):2350-2360.

7. Ciuffreda D, et al. Polyfunctional HCV-specific $T$-cell responses are associated with effec- 
tive control of HCV replication. Eur J Immunol. 2008;38(10):2665-2677.

8. Wherry EJ. T cell exhaustion. Nat Immunol. 2011;12(6):492-499.

9. Betts MR, et al. HIV nonprogressors preferentially maintain highly functional HIV-specific CD8+ T cells. Blood. 2006;107(12):4781-4789.

10. Frebel H, Richter K, Oxenius A. How chronic viral infections impact on antigen-specific T-cell responses. Eur J Immunol. 2010;40(3):654-663.

11. Mueller SN, Ahmed R. High antigen levels are the cause of $\mathrm{T}$ cell exhaustion during chronic viral infection. Proc Natl Acad Sci U S A. 2009; 106(21):8623-8628.

12. Han S, Asoyan A, Rabenstein H, Nakano N, Obst R. Role of antigen persistence and dose for CD4+ T-cell exhaustion and recovery. Proc Natl Acad Sci U S A. 2010;107(47):20453-20458.

13. Barber DL, et al. Restoring function in exhausted CD8 T cells during chronic viral infection. Nature. 2006;439(7077):682-687.

14. Day CL, et al. PD-1 expression on HIV-specific $\mathrm{T}$ cells is associated with T-cell exhaustion and disease progression. Nature. 2006;443(7109):350-354.

15. Trautmann L, et al. Upregulation of PD-1 expression on HIV-specific CD8+ T cells leads to reversible immune dysfunction. Nat Med 2006;12(10):1198-1202.

16. Blackburn SD, et al. Coregulation of CD8+ $\mathrm{T}$ cell exhaustion by multiple inhibitory receptors during chronic viral infection. Nat Immunol. 2009;10(1):29-37.

17. Jin HT, et al. Cooperation of Tim-3 and PD- 1 in CD8 T-cell exhaustion during chronic viral infection. Proc Natl Acad Sci U S A. 2010;107(33):14733-14738.

18. Darrah PA, et al. Multifunctional TH1 cells define a correlate of vaccine-mediated protection against Leishmania major. Nat Med. 2007;13(7):843-850.

19. Sallusto F, Geginat J, Lanzavecchia A. Central memory and effector memory $\mathrm{T}$ cell subsets: function, generation, and maintenance. Annu Rev Immu nol. 2004;22:745-763.

20. Intlekofer AM, et al. Effector and memory CD8+ $\mathrm{T}$ cell fate coupled by T-bet and eomesodermin. Nat Immunol. 2005;6(12):1236-1244.

21. Zhao DM, et al. Constitutive activation of Wnt signaling favors generation of memory CD8 T cells. Immunol. 2009;184(3):1191-1199.

22. Kohlmeier JE, et al. Inflammatory chemokine receptors regulate $\mathrm{CD} 8(+) \mathrm{T}$ cell contraction and memory generation following infection. J Exp Med. 2010;208(8):1621-1634.
23. Kurachi M, et al. Chemokine receptor CXCR3 facilitates CD8(+) T cell differentiation into short-lived effector cells leading to memory degeneration. J Exp Med. 2011;208(8):1605-1620.

24. Haining WN, Wherry EJ. Integrating genomic signatures for immunologic discovery. Immunity. 2010;32(2):152-161.

25. Wherry EJ, et al. Molecular signature of CD8+ $\mathrm{T}$ cell exhaustion during chronic viral infection. Immunity. 2007;27(4):670-684.

26. Quigley M, et al. Transcriptional analysis of HIVspecific CD8+ T cells shows that PD- 1 inhibits $T$ cell function by upregulating BATF. Nat Med. 2010; 16(10):1147-1151.

27. Francisco LM, Sage PT, Sharpe AH. The PD-1 pathway in tolerance and autoimmunity. Immunol Rev. 2010;236:219-242.

28. Oelke M, Maus MV, Didiano D, June CH, Mackensen A, Schneck JP. Ex vivo induction and expansion of antigen-specific cytotoxic T cells by HLA-Igcoated artificial antigen-presenting cells. Nat Med 2003;9(5):619-624.

29. Chiu YL, Schneck JP, Oelke M. HLA-Ig based artificial antigen presenting cells for efficient ex vivo expansion of human CTL. $J$ Vis Exp. 2011;(50):pii:2801.

30. Dong C, Davis RJ, Flavell RA. MAP kinases in the immune response. Annu Rev Immunol. 2002;20:55-72.

31. Cabrita MA, Christofori G. Sprouty proteins, masterminds of receptor tyrosine kinase signaling. Angiogenesis. 2008;11(1):53-62.

32. Hanafusa H, Torii S, Yasunaga T, Nishida E. Sprouty1 and Sprouty2 provide a control mechanism for the Ras/MAPK signalling pathway. Nat Cell Biol. 2002;4(11):850-858.

33. Yusoff $\mathrm{P}$, et al. Sprouty 2 inhibits the Ras/MAP kinase pathway by inhibiting the activation of Raf. J Biol Chem. 2002;277(5):3195-3201.

34. Akbulut S, et al. Sprouty proteins inhibit receptor-mediated activation of phosphatidylinositol-specific phospholipase C. Mol Biol Cell. 2010;21(19):3487-3496

35. Migueles SA, et al. Defective human immunodeficiency virus-specific CD8+ T-cell polyfunctionality, proliferation, and cytotoxicity are not restored by antiretroviral therapy. J Virol. 2009; 83(22):11876-11889.

36. Joshi NS, Kaech SM. Effector CD8 T cell development: a balancing act between memory cell potential and terminal differentiation. J Immunol. 2008;180(3):1309-1315

37. Zhou X, Yu S, Zhao DM, Harty JT, Badovinac VP,
Xue HH. Differentiation and persistence of memory CD8(+) T cells depend on $\mathrm{T}$ cell factor 1 . Immunity. 2010;33(2):229-240.

38. Lee JS, et al. Recruitment of Sprouty1 to immune synapse regulates T cell receptor signaling. J Immunol. 2009;183(11):7178-7186.

39. Collins S, et al. Regulation of CD4(+) and CD8(+) Effector Responses by Sprouty-1. PLoS One. 2012;7(11):e49801.

40. Takata H, Naruto T, Takiguchi M. Functional heterogeneity of human effector CD8+ T cells. Blood. 2012;119(6):1390-1398.

41. Golden-Mason L, Palmer B, Klarquist J, Mengshol JA, Castelblanco N, Rosen HR. Upregulation of PD-1 expression on circulating and intrahepatic hepatitis C virus-specific CD8+ T cells associated with reversible immune dysfunction. J Virol. 2007;81(17):9249-9258.

42. Blackburn SD, Shin H, Freeman GJ, Wherry EJ. Selective expansion of a subset of exhausted CD8 T cells by alphaPD-L1 blockade. Proc Natl Acad Sci US A. 2008;105(39):15016-15021.

43. Ndhlovu ZM, Oelke M, Schneck JP, Griffin DE. Dynamic regulation of functionally distinct virus-specific T cells. Proc Natl Acad Sci U S A. 2010;107(8):3669-3674.

44. Roederer M, Nozzi JL, Nason MC. SPICE: exploration and analysis of post-cytometric complex multivariate datasets. Cytometry A. 2011;79(2):167-174.

45. Huang da W, Sherman BT, Lempicki RA. Systematic and integrative analysis of large gene lists using DAVID bioinformatics resources. Nat Protoc. 2009;4(1):44-57.

46. Subramanian A, et al. Gene set enrichment analysis: a knowledge-based approach for interpreting genome-wide expression profiles. Proc Natl Acad Sci US A. 2005;102(43):15545-15550.

47. Chow S, Patel H, Hedley DW. Measurement of MAP kinase activation by flow cytometry using phospho-specific antibodies to MEK and ERK: potential for pharmacodynamic monitoring of signal transduction inhibitors. Cytometry. 2001;46(2):72-78

48. Lecoeur H, Fevrier M, Garcia S, Riviere Y, Gougeon ML. A novel flow cytometric assay for quantitation and multiparametric characterization of cellmediated cytotoxicity. J Immunol Methods. 2001; 253(1-2):177-187.

49. Lamason RL, Kupfer A, Pomerantz JL. The dynamic distribution of CARD11 at the immunological synapse is regulated by the inhibitory kinesin GAKIN. Mol Cell. 2010;40(5):798-809. 